DOI: http://doi.org/10.22585/hospdomic.v5i2.127

\title{
Manejo de la enfermedad tromboembólica venosa en la hospitalización a domicilio: ¿¿Qué pacientes atendemos y cuál es su perfil de riesgo?
}

Management of Venous Trhomboembolic Disease in Home Hospitalization: Which Patients Do We Treat and What Is Their Risk Stratification?

David Sánchez Fabra' (1) 0000-0002-6763-9963

Nuria Saura Blasco ${ }^{2}$ (1) 0000-0002-6809-589X

Raquel Tascón Rodriguez ${ }^{2}$ (1) 0000-0003-0193-1555

Adriana Ger Buil' (1) 0000-0003-2701-4376

Ana María Camón Pueyo' (1) 0000-0001-9924-4037

Leire Huete Álava' (1) 0000-0002-2289-715X

1. Hospital Reina Sofía, Tudela, España.

2. Hospital Universitario Lozano Blesa, Zaragoza, España.

Correspondencia/Correspondence

David Sánchez Fabra

davidsanchezfabra@gmail.com

Recibido/Received

01.12 .2020

Aceptado/Accepted

31.01 .2021

Conflicto de Intereses/Competing interest

Sin conflictos de intereses.
Financiación/Funding

El trabajo no recibió ningún tipo de financiación.

Contribuciones de autoría/Author contributions

El autor y las autoras declaran que han contribuido por igual en la realización de este trabajo.

Agradecimientos/Acknowledgments

No los hay.

CÓMO CITAR ESTE TRABAJO | HOW TO CITE THIS PAPER

Sánchez-Fabra D, Saura-Blasco N, Tascón-Rodríguez R, Ger-Buil A, Camón-Pueyo AM, Huete-Álava L. Manejo de la enfermedad tromboembólica venosa en la hospitalización a domicilio: ¿¿Qué pacientes atendemos y cuál es su perfil de riesgo?. Hosp Domic. 2021;5(2):79-87. 


\section{RESUMEN}

Introducción: Las Guías de Práctica Clínica recogen el mejor manejo de la Enfermedad Tromboembólica Venosa (ETEV) en función de la estratificación del riesgo en cuanto a ingreso o tratamiento ambulatoria. La utilidad de la Hospitalización a Domicilio (HAD) en esta enfermedad es poco conocida.

Métodos: Realizamos un estudio descriptivo y comparativo de todos los pacientes admitidos en HAD por ETEV durante dos años, emparejándolos con otro grupo de pacientes de hospitalización convencional (HC). Analizamos también el destino de los pacientes (HAD o HC) en función de la estratificación del riesgo.

Resultados: Obtuvimos una muestra de $n=76$ pacientes. La estancia hospitalaria fue menor en el grupo de HAD. No hubo diferencias estadísticamente significativas en el resto de variables. El 22,9\% de los pacientes con riesgo bajo de mortalidad ingresaron.

Conclusión: La estratificación del riesgo o las características clínicas de los pacientes con ETEV no tuvieron relevancia frente a la elección del destino en régimen de HAD, pudiendo haber primado más los criterios generales de ingreso en HAD (preferencias del paciente, soporte domiciliario adecuado, o el manejo de otras comorbilidades).

Palabras clave: Enfermedad tromboembólica Venosa; Tromboemobolismo Pulmonar; Trombosis; Venosa Profunda; Hospitalización a Domicilio; Medicina Interna.

\section{ABSTRACT}

Introduction: Clincal Practice Guidelines show the best management of Venous Thromboembolic Disease (VTE) based on risk stratification in terms of admission or outpatient treatment. The utility of Home Hospitalization $(\mathrm{HH})$ in this disease is poorly understood.

Methods: We conducted a descriptive and comparative study of all patients admitted at $\mathrm{HH}$ for VTE for two years, matching them with another group of conventional hospitalization $(\mathrm{CH})$ patients. We also analyzed the destination of the patients $(\mathrm{HH}$ or $\mathrm{CH})$ based on their risk stratification.

Results: We obtained a sample of $n=76$ patients. The hospital length of stay was shorter in the HAD group. There were no statistically significant differences in the rest of the variables. $22.9 \%$ of patients with low risk of mortality were admitted at $\mathrm{CH}$ or $\mathrm{HH}$.

Conclusion: Risk stratification or clinical characteristics of patients with VTE were not relevant when it came to the choice of destination under the $\mathrm{HH}$ regimen. General criteria for admission to $\mathrm{HH}$ (patient preferences, adequate home support, or management other comorbidities) may have prevailed more in order to take that decision.

Keywords: Venous thromboembolic disease; Pulmonary Embolism; Deep venous thrombosis; Home Hospitalization; Internal Medicine. 


\section{INTRODUCCIÓN}

La Enfermedad Tromboembólica Venosa (ETEV) es una patología frecuente que incluye tanto el Tromboembolismo Pulmonar (TEP) como la Trombosis Venosa Profunda (TVP). Aunque su conocimiento y manejo ha mejorado a lo largo de los años, se ha producido un incremento de la incidencia, situándose en 32,69/100.000 habitantes/año en España ${ }^{(1)}$. En las guías de práctica clínica (GPC) se recoge el manejo más adecuado de tratamiento del paciente (ingresado/ambulatorio) en función de las escalas pronosticas y de riesgo ${ }^{(2-4)}$; sin embargo, la utilidad de la hospitalización a domicilio (HAD) en la ETEV no es muy conocida.

Hay suficiente evidencia para considerar seguro el manejo ambulatorio tanto de la TVP ${ }^{(5,6)}$ como del TEP en pacientes seleccionados ${ }^{(7)}$. Un estudio demostró que también en HAD existe margen para tratar el TEP durante todo el curso de la enfermedad ${ }^{(8)}$. Pese a la evidencia disponible, son varios los autores que reconocen que en la práctica real no siempre el manejo de los pacientes con ETEV se adecúa a las GPC $^{(9)}$ y que un porcentaje reseñable de candidatos a manejo ambulatorio son ingresados o pasan demasiado tiempo en el hospital(9).

Dada la escasa bibliografía sobre la ETEV en la HAD, en el presente estudio pretendemos determinar las características de los pacientes ingresados por ETEV tanto en hospitalización convencional (HC) como en HAD, así como la adecuación de la modalidad de ingreso en función de sus escalas pronosticas.

\section{MÉTODOS}

Se seleccionaron en los registros informáticos todos los pacientes mayores de 18 años con diagnóstico al alta de ETEV (TEP o TVP) en el Hospital Reina Sofía de Tudela entre el 1/1/18 y el 31/12/19. En primer lugar, se detectaron aquellos del grupo de HAD dado el menor tamaño muestral y, tras ello, se realizó un muestreo simple aleatorio en el grupo de pacientes de HC para seleccionar una muestra idéntica.

Se registraron variables demográficas, de comorbilidades, clínicas, analíticas, de estratificación del riesgo, de tratamiento, de complicaciones y de resultados. Los grupos estudiados fueron el de pacientes que ingresaron en algún momento en HAD (directamente desde urgencias o desde planta) y el de HC (los que cumplieron todo el ingreso en el hospital). Se excluyeron los casos dados de alta desde urgencias. En cuanto a la estratificación del riesgo se empleó la escala PESI (Pulmonary Embolism Severity Index) y las escalas de las GPC (riesgo bajo, intermedio-bajo, intermedio-alto y alto) (2).

Para el análisis de las variables cualitativas se utilizó sus frecuencias y procentajes y, para el contraste de hipótesis, el test de Chi cuadrado (con la corrección de Fisher cuando fue necesario). Las variables cuantitativas se expresaron mediante las medidas de tendencia central: media, mediana y cuartiles y para la estimación la prueba de la $U$ de Mann Withney dado el carácter no paramétrico de los datos. El análisis estadístico se efectuó mediante el programa estadístico SPSS ${ }^{\circledR}$ (Statistical Package for the Social Sciences) versión 15 para Windows.

Se obtuvo el consentimiento del comité ético de Navarra. 


\section{RESULTADOS}

Obtuvimos una muestra de 76 pacientes (38 en cada grupo). Sus características principales vienen detalladas en la tabla 1.

\begin{tabular}{|c|c|}
\hline Variable & Resultado \\
\hline Ingreso en $\mathrm{HAD}, \mathrm{n}(\%)$ & $38(50)$ \\
\hline Ingreso directamente en HAD ( $\mathrm{n}=38) ; \mathrm{n}(\%)$ & $22(57,9)$ \\
\hline Sexo mujer; n (\%) & $39(51,3)$ \\
\hline Edad; media (p25/mediana/p75) & $71,8(59 / 75,5 / 85)$ \\
\hline TEP; n (\%) & $35(46,1)$ \\
\hline TVP; n (\%) & $50(65,8)$ \\
\hline TVP EESS; n (\%) & $3(6)$ \\
\hline TVP EEII; n (\%) & $44(88)$ \\
\hline TVP EEII proximal; n (\%) & $33(66)$ \\
\hline TVP EEll distal; n (\%) & $37(74)$ \\
\hline Índice de Charlson; media (p25/mediana/p75) & $3,5(0 / 4 / 5,75)$ \\
\hline Insuficiencia cardíaca; n (\%) & $10(13,2)$ \\
\hline Demencia; n (\%) & $3(3,3)$ \\
\hline EPOC; n (\%) & $12(15,8)$ \\
\hline ETEV previa; n (\%) & $16(21,1)$ \\
\hline Cáncer activo; n (\%) & $6(7,9)$ \\
\hline Anticoagulación previa; n (\%) & $3(3,9)$ \\
\hline Etiología provocada; n (\%) & $21(27,6)$ \\
\hline
\end{tabular}




\begin{tabular}{|c|c|}
\hline Variable & Resultado \\
\hline \multicolumn{2}{|l|}{ Tratamientos: } \\
\hline Con HPBM; n (\%) & $76(100)$ \\
\hline Con AVK; n (\%) & $63(82,9)$ \\
\hline Con ACOD; n (\%) & $1(1,3)$ \\
\hline Con HNF; n (\%) & $2(2,6)$ \\
\hline Trombectomía; n (\%) & $1(1,3)$ \\
\hline Fibrinólisis; n (\%) & 0 \\
\hline Reingreso desde HAD al hospital ( $\mathrm{n}=38)$; $\mathrm{n}(\%)$ & $2(5,3)$ \\
\hline Estancia en el hospital ( $n=52)$; media (p25/mediana/p75) & $5,5(3 / 5 / 7)$ \\
\hline Estancia en HAD; media (p25/mediana/p75) & $7,5(5 / 7 / 9)$ \\
\hline Estancia total; media (p25/mediana/p75) & $7,6(5 / 6 / 9)$ \\
\hline Valor PESI ( $n=35)$; media ( $\mathrm{p} 25 /$ mediana/p75) & $118(89 / 110 / 138)$ \\
\hline Clase I; n (\%) & 0 \\
\hline Clase II; n (\%) & $8(22,9)$ \\
\hline Clase III; n (\%) & $9(25,7)$ \\
\hline Clase IV; n (\%) & $6(17,1)$ \\
\hline Clase V; n (\%) & $12(34,3)$ \\
\hline Algún evento adverso; n (\%) & $9(11,8)$ \\
\hline Sangrado intrahospitalario; n (\%) & $4(5,3)$ \\
\hline Recurrencia; n (\%) & 0 \\
\hline Exitus intrahospitalario; $\mathrm{n}(\%)$ & $3(3,9)$ \\
\hline 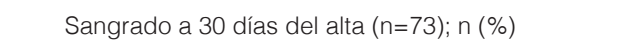 & $1(1,4)$ \\
\hline
\end{tabular}




\begin{tabular}{|l|l|}
\hline Variable & Resultado \\
\hline Recurrencia a 30 días del alta $(\mathrm{n}=73) ; \mathrm{n}(\%)$ & 0 \\
\hline Reingreso a 30 días del alta $(\mathrm{n}=73) ; \mathrm{n}(\%)$ & $2(2,7)$ \\
\hline Exitus a 30 días del alta $(\mathrm{n}=73) ; \mathrm{n}(\%)$ & $1(1,4)$ \\
\hline
\end{tabular}

Siglas: HAD, Hospitalización a Domicilio; p25/p75, cuartiles; TEP, Tromboembolismo Pulmonar; TVP, Trombosis Venosa Profunda; EEII, Extremidades Inferiores; EESS, Extremidades Superiores; EPOC, Enfermedad Pulmonar Obstructiva Crónica; ETEV, Enfermedad Tromboembólica Venosa; HBPM, Heparina de Bajo Peso Molecular; AVK, Anti-Vitamina K; ACOD, Anticoagulantes de Acción Directa; HNF, Heparina No Fraccionada; PESI, Pulmonary Embolism Severity Index. Aclaraciones: Salvo especificación de lo contrario, la n es de 76 pacientes. Excepciones: $(n=35)$, para los análisis de PESI y estratificación del riesgo solo se utilizaron los casos de TEP, excluyéndose TVP; $(n=73)$, para dicho análisis se excluyeron los pacientes que murieron durante el ingreso índice.

En el grupo de HAD la estancia hospitalaria fue menor (3,9 días frente a 6,2 días, $p=0,006$ ), pero la estancia total fue mayor $(9,1$ frente a $6,2, p=0,004)$. No se encontraron diferencias significativas en el resto de variables (edad, comorbilidades o gravedad por PESI).

De los 38 pacientes que estuvieron ingresados en HAD, $22(57,9 \%)$ fueron directamente desde urgencias. La presencia de TEP fue menos frecuente en ellos ( $n=4,18,2 \%$ respecto a $n=11,69,8 \%$ en el grupo que pasó por planta; $p=0,002$ ), siendo por el contrario más frecuente la presencia de $\operatorname{TVP}(n=19,86,4 \%$ respecto a $n=8,50 \% ; p=0,015)$. La estancia total fue menor en los pacientes que ingresaron directamente en $\operatorname{HAD}(7,5$ días respecto a 11,$1 ; p=0,032)$, encontrando una tendencia a ser más mayores (edad 76,6 frente a 68,1 años; $p=0,058$ ).

En cuanto al manejo de las escalas de riesgo en el TEP, la distribución de los pacientes y sus características vienen detalladas en la tabla 2.

Tabla 2. Características de los pacientes con Tromboembolismo Pulmonar (TEP) según su estratificación de riesgo y manejo tras el diagnóstico

\begin{tabular}{|c|l|l|l|l|}
\hline Estratificación del riesgo en TEP $(\mathrm{n}=35)$ & Bajo & $\begin{array}{l}\text { Intermedio- } \\
\text { Bajo }\end{array}$ & $\begin{array}{l}\text { Intermedio- } \\
\text { Alto }\end{array}$ & Alto \\
\hline Total, $\mathrm{n}(\%)$ & $8(22,9)$ & $16(45,7)$ & $7(20)$ & $4(11,4)$ \\
\hline $\begin{array}{c}\text { Ingreso solo en planta }(\mathrm{n}=20), \mathrm{n}(\%) \\
\begin{array}{c}\text { Ingreso en HAD pasando por planta } \\
(\mathrm{n}=11), \mathrm{n}(\%)\end{array}\end{array}$ & $6(30)$ & $7(35)$ & $5(25)$ & $2(10)$ \\
\hline $\begin{array}{c}\text { Ingreso en HAD directamente }(\mathrm{n}=4), \mathrm{n} \\
(\%)\end{array}$ & 0 & $6(54,5)$ & $1(9,1)$ & $2(18,2)$ \\
\hline \begin{tabular}{c} 
Edad (media/RI) \\
\hline Índice de Charlson (media/RI)
\end{tabular} & $65,6(26)$ & $72,1(24)$ & $83,4(15)$ & $85(17)$ \\
\hline
\end{tabular}




\begin{tabular}{|l|l|l|l|l|}
\hline Estratificación del riesgo en TEP $(\mathbf{n = 3 5 )}$ & Bajo & $\begin{array}{l}\text { Intermedio- } \\
\text { Bajo }\end{array}$ & $\begin{array}{l}\text { Intermedio- } \\
\text { Alto }\end{array}$ & Alto \\
\hline Estancia hospitalaria (media/RI) & $5,75(4)$ & $5,46(3)$ & $8(14)$ & $9,75(12)$ \\
\hline Estancia en HAD (media/RI) & $7(0)$ & $10,14(7)$ & $15(0)$ & $11,15(0)$ \\
\hline Estancia total (media/RI) & $7,5(4)$ & $9(6)$ & $12,6(12)$ & $15,5(14)$ \\
\hline
\end{tabular}

Escalas de riesgo de la "2019 ESC Guidelines for the diagnosis and management of acute pulmonaryembolism developed in collaboration with the European Respiratory Society (ERS)": Bajo riesgo (estabilidad hemodinámica, clase PESI<III y sin datos de disfunción del ventrículo derecho o elevación de marcadores de daño miocárdico), Riesgo intermedio-bajo (estabilidad hemodinámica, clase PESI III-V con datos de disfunción del ventrículo derecho o elevación de marcadores de daño miocárdico), Riesgo intermedio-alto (estabilidad hemodinámica, clase PESI III-V con datos de disfunción del ventrículo derecho y elevación de marcadores de daño miocárdico) o Alto riesgo (inestabilidad hemodinámica, clase PESI III-V con datos de disfunción del ventrículo derecho y elevación de marcadores de daño miocárdico)(2). Siglas: TEP, Tromboembolismo Pulmonar; HAD, Hospitalización a Domicilio; RI, Rango Intercuartílico.

\section{DISCUSIÓN}

Encontramos que los pacientes y el manejo de la ETEV en HAD y HC son muy similares. Un porcentaje importante de pacientes con TEP y riesgo bajo, que podrían ser candidatos a tratamiento ambulatorio $^{(2)}$, son ingresados $(22,9 \%)$.

El paciente típico que presenta ETEV en nuestro medio es una persona de unos 70 años y escasa comorbilidad, correspondiendo en casi la mitad de los casos $(46,1 \%)$ a TEP. Los resultados adversos (reingreso desde HAD, sangrado, recurrencia, exitus o reingreso tras el alta) en ambos fueron infrecuentes, sin que existieran diferencias significativas y siendo similares a los observados en otros trabajos. En un estudio sobre el manejo de la TVP en pacientes hospitalizados vs ambulatorios encontraron tasas de recurrencia, sangrado o muerte menores al 1\% en ambos grupos, objetivando un menor riesgo de hemorragia mayor (OR 0,4; IC 95\% 0,1-1) y de muerte (OR 0,2; IC95\% $0,1-0,7)$ en los pacientes ambulatorios ${ }^{(5)}$. Rodríguez-Cerrillo et al, comparando pacientes con TEP (30 en HAD, 31 en $\mathrm{HC}$ ), publicaron que ninguno de ellos presentó hemorragia mayor, recurrencia o muerte $^{(7)}$. Tampoco ocurrieron hemorragias mayores o muertes en el grupo de 21 pacientes con TEP tratados en HAD en el estudio de Lui et al( ${ }^{(8)}$. En el análisis sobre práctica clínica real de Dentali et al, ( $n=2218 \mathrm{ETEV} ; 34,5 \%$ TEP con o sin TVP) las tasas de recurrencia, hemorragia mayor y mortalidad fueron bajas a 90 días de seguimiento $(0,63 \%, 0,81 \% \text { y } 2,16 \%)^{(10)}$. Si bien consideramos que las diferencias encontradas en cuanto a la estancia podrían deberse a las dinámicas asistenciales del trabajo en HAD (posible selección de pacientes que requirieran un control hospitalario más largo), creemos que la similitud entre ambos grupos en el resto de variables podría implicar que las decisiones de manejo en HAD o HC se basen en los criterios generales de ingreso en HAD (adecuado soporte domiciliario, cuidador disponible o preferencias del paciente) y no en las características específicas de la enfermedad o su estratificación de riesgo.

En lo referente a la adecuación a las GPC en cuanto a la estratificación del riesgo en pacientes con TEP, observamos que un $22,9 \%$ de los ingresos tenían riesgo bajo de mortalidad, lo que podría haber motivado un manejo ambulatorio inicial. De ellos, además, solo 2 (25\%) pasaron parte del ingreso en régimen de HAD. Consideremos que quizá existe cierto margen de mejora en este aspecto. Dentali et al concluyeron que el $53,7 \%$ de las TVP y el $17 \%$ de los TEP son tratados am- 
bulatoriamente sin que la estratificación de riesgo por PESI afectara ni a la decisión de ingreso ni a la duración del mismo ${ }^{(10)}$.

Nuestro estudio tiene algunas limitaciones. Fue unicéntrico y con una muestra pequeña, lo que restaría potencia estadística. El carácter observacional de revisión de historias clínicas podría implicar también una pérdida de información valiosa respecto a algunos motivos de ingreso. Sin embargo, creemos haber retratado al paciente real con ETEV que es atendido en HAD y en HC. Por otro lado, son muy pocos los trabajos publicados que estudian las características y manejo de la ETEV en HAD ya que la mayoría, hasta la fecha, se han centrado en analizar las diferencias entre HC y tratamiento ambulatorio. No nos es ajeno que parte de la bibliografía referida ${ }^{(5,9,10)}$ extrapola los datos de pacientes ambulatorios, sin que pueda considerarse que su manejo equivalga al de la HAD como sí pudiera hacerse con el resto de la bibliografía ${ }^{(7,8)}$. Es por ello que consideramos que nuestro trabajo aporta luz a la posibilidad de atender los pacientes con ETEV en régimen de HAD, un tema muy poco conocido.

En la ETEV, y pese a que las GPC y la mayoría de la bibliografía evalúa el manejo ambulatorio vs hospitalario de los pacientes, existe un amplio margen de actuación para la HAD. Se hace necesario un mayor conocimiento en este campo para saber reconocer qué pacientes podrían beneficiarse de este tipo de ingreso, lo que se traduciría en una mejora de la asistencia y una reducción de los costes hospitalarios.

\section{BIBLIOGRAFÍA}

1. De Miguel-Diez J, Jiménez-Garcia R, Jiménez D, Monreal M, Guijarro R, Otero R, et al. Trends in hospital admissions for pulmonary embolism in Spain from 2002 to 2011. Eur Respir J. 2014;44(4):942-50. DOI: 10.1183/09031936.00194213

2. Konstantinides SV, Meyer G, Becattini C, Bueno H, Geersing GJ, Harjola VP, et al; ESC Scientific Document Group. 2019 ESC Guidelines for the diagnosis and management of acute pulmonary embolism developed in collaboration with the European respiratory society (ERS). Eur Heart J. 2020;41(4):543-603. DOI: 10.1093/eurheartj/ehz405

3. Mazzolai L, Aboyans V, Ageno W, Agnelli G, Alatri A, Bauersachs R, et al. Diagnosis and management of acute deep vein thrombosis: A joint consensus document from the European Society of Cardiology working groups of aorta and peripheral vascular diseases and pulmonary circulation and right ventricular function. Eur Heart J. 2018;39(47):4208-18. DOI: 10.1093/eurheartj/ ehx003

4. Corrigendum to: Diagnosis and management of acute deep vein thrombosis: a joint consensus document from the european society of cardiology working groups of aorta and peripheral circulation and pulmonary circulation and right ventricular function. Eur Heart J. 2018;39(15):1316. DOI: 10.1093/eurheartj/ehx164

5. Lozano F, Trujillo-Santos J, Barrón M, Gallego P, Babalis D, Santos M, et al. Home versus inhospital treatment of outpatients with acute deep venous thrombosis of the lower limbs. J Vasc Surg. 2014;59(5):1362-7.e1. DOI: 10.1016/j.jvs.2013.11.091

6. Othieno R, Okpo E, Forster R. Home versus in-patient treatment for deep vein thrombosis. Cochrane Database Syst Rev. 2018; 1(1):CD003076. DOI: 10.1002/14651858.CD003076.pub3 
7. Rodríguez-Cerrillo M, Alvarez-Arcaya A, Fernández-Díaz E, Fernández-Cruz A. A prospective study of the management of non-massive pulmonary embolism in the home. Eur $\mathrm{J}$ Intern Med. 2009;20(6):598-600. DOI: 10.1016/j.ejim.2009.04.003

8. Lui B, Tran A, Montalto M. Treatment of patients with pulmonary embolism entirely in hospital in the home. Aust Fam Physician. 2007;36(5):381-4.

9. Caprini JA, Tapson VF, Hyers TM, Waldo AL, Wittkowsky AK, Friedman R, et al; NABOR Steering Committee. Treatment of venous thromboembolism: Adherence to guidelines and impact of physician knowledge, attitudes, and beliefs. J Vasc Surg. 2005;42(4):726-33. DOI: 10.1016/j. jvs.2005.05.053

10. Dentali F, Di Micco G, Giorgi Pierfranceschi M, Gussoni G, Barillari G, Amitrano M, et al. Rate and duration of hospitalization for deep vein thrombosis and pulmonary embolism in real-world clinical practice. Ann Med. 2015;47(7):546-54. DOI: 10.3109/07853890.2015.1085127 